\title{
Pulgarización del Índice en el Tratamiento de la Hipoplasia de Pulgar - Técnica Quirúrgica
}

\section{Index Finger Pollicization in the Treatment of Thumb Hypoplasia - Surgical Technique}

\author{
Manuel Mendez ${ }^{1,2}$ Elias Concha1,3,4 Catalina Parot ${ }^{1,4}$ Marcia Carrasco ${ }^{1,5}$
}

\footnotetext{
${ }^{1}$ Departamento de Ortopedia, Extremidad Superior, Instituto Teletón Santiago, Chile

${ }^{2}$ Departamento Ortopedia, Clínica las Condes, Santiago Chile

${ }^{3}$ Departamento Ortopedia, Clínica Meds, Santiago, Chile

${ }^{4}$ Servicio de Traumatología, Hospital Luis Calvo Mackenna, Santiago, Chile

5 Servicio Ortopedia y Cirugía Infantil, Hospital de Carabineros,

Santiago, Chile
}

Rev Chil Ortop Traumatol 2017;58:95-99.

\begin{abstract}
Address for correspondence Manuel Mendez, MD, Departamento de Ortopedia, Clínica Las Condes, Estoril 450, Las Condes, Santiago, Chile (e-mail: mmendez@clinicalascondes.cl).
\end{abstract}

\section{Resumen \\ Palabras Claves \\ - pulgarización \\ - hipoplasia de pulgar}

\section{Abstract}

Keywords

- index pollicization

- congenital thumb hypoplasia
La pulgarización del dedo índice permite reconstruir la pinza de la mano. Fue ampliamente realizada para una reconstrucción de una pérdida de pulgar en condiciones de trauma. Sin embargo, hoy por hoy, es la técnica de elección para las hipoplasias congénitas de pulgar. En los niños permite crear una pinza, especialmente para objetos grandes y entrega una apariencia más normal de la mano. Es una técnica demandante, pero con pasos que están bien definidos y perfeccionados, que al ser realizados de forma cuidadosa, reducen al mínimo sus complicaciones.

The pollicization of the index finger allows reconstructing the hand's ability to pinch. It has been broadly used to address traumatic loss of the thumb. However today it is the procedure of choice for severe congenital thumb hypoplasia. It allows children to pinch, particularly large objects, it also gives the hand a more normal appearance. It is a demanding procedure, with a series of technical steps that are well defined and perfected, such that if they are performed in a careful way, complications are minimized.

\section{Introducción}

La pulgarización del índice es una las más desafiantes y gratificantes cirugías de la mano, que combina técnicas de ortopedia, cirugía plástica y la participación de un grupo multidiciplinario.

Los objetivos de esta técnica son básicamente dos. Primero, otorga a la mano la posibilidad de tener una pinza para tomar objetos grandes. Y segundo, esta técnica entrega una mano de apariencia "más normal."

La transferencia del dedo índice para el tratamiento de la ausencia de pulgar, se remonta a la década de los 40 del siglo pasado, y en su inicio, esa fue propuesta para el tratamiento en la amputación traumática del pulgar. Hilgenfeldt recomendó la pulgarizacion del dedo medio. ${ }^{1}$ Gosset describió la técnica con el dedo índice con colgajos para asegurar la creación del primer espacio. $^{2}$

Pero son los trabajos de Littler en 1953, los que sientan las bases de la técnica que hoy conocemos, permitiendo tener un colgajo neurovascular en isla (dedo índice), y entrega los conceptos de las transferencias musculares que permiten crear los "nuevos" músculos de la región tenar para potenciar los resultados funcionales de la cirugía. $^{3}$ received

May 22, 2017

accepted

September 12, 2017

published online

December 6, 2017
DOI https://doi.org/

10.1055/s-0037-1607287. ISSN 0716-4548.
Copyright (e 2017 by Thieme Revinter

Publicações Ltda, Rio de Janeiro, Brazil
License terms

(c) $(1) \$$ 
La realización de esa técnica en lesiones congénitas, tiene su gran exponente y refinamientos de la técnica, en los trabajos de Riordan y Buck-Gramcko. Ese último realizo más de 450 pulgarizaciones y 200 de ellas fueron en malformaciones congénitas producidas por el uso de la talidomida entre los años 1959-1962. ${ }^{4}$

\section{Indicación}

El presente trabajo aborda la pulgarización del índice, en el contexto de las hipoplasias del pulgar.

Según la clasificación de Blauth ${ }^{5}$ en las hipoplasias de pulgar se incluyen 5 tipos. La misma fue más tarde modificada por Manske y Carroll, los que dividieron la tipo III en A y B, según la estabilidad o no de la trapecio-metacarpiana. ${ }^{6}$ Hoy por hoy, la pulgarización del índice está indicada para el tratamiento de las hipoplasia tipo IIIB, IV, y V.

Kozin tiene una interesante comunicación en donde muestra otras indicaciones para esa técnica que incluye macrodactilia, mano como espejo y polidactilias. ${ }^{7}$

\section{Técnica quirúrgica}

Los principios de la pulgarización incluyen una cuidadosa planificación de los colgajos, disección de estructuras vasculares y nerviosas, resección ósea, creación de nuevas articulaciones, y un complejo posicionamiento en tres planos del neo pulgar.

1. Diseño y incisión de la piel. El diseño del colgajo incluye un colgajo de base y vértice proximal en el dorso del índice, y que por lateral y volar alcanza la región media-distal de la F1 del índice. Es este nivel de disección el que define la apariencia de "pulgar," ya que si el diseño es muy cercano a la base del índice, puede dar la apariencia de un índice colocado al lado de la mano. Otro aspecto importante es el nivel anterior o lateral que pueda alcanzar la incisión proximal en la región tenar, ya que puede condicionar el nivel de antepulsión que pueda tener el "pulgar" (-Figs. 1 y 2).

2. Elevación de colgajo dorsal y volar. La elevación del colgajo dorsal es el que permite identificar las venas dorsales del índice que será transportado. Es frecuente identificar dos

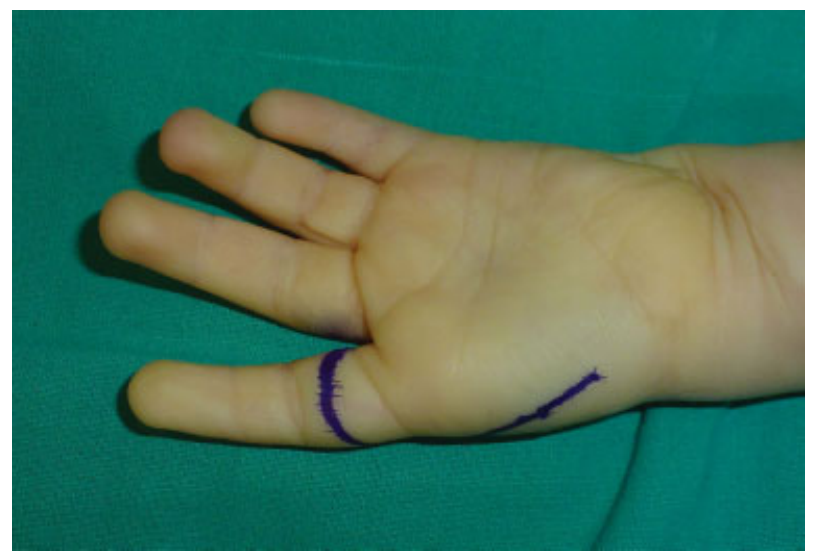

Fig. 1 Diseños de los colgajos. El nivel del colgajo a nivel de la F1, puede influir en la apariencia del nuevo pulgar.

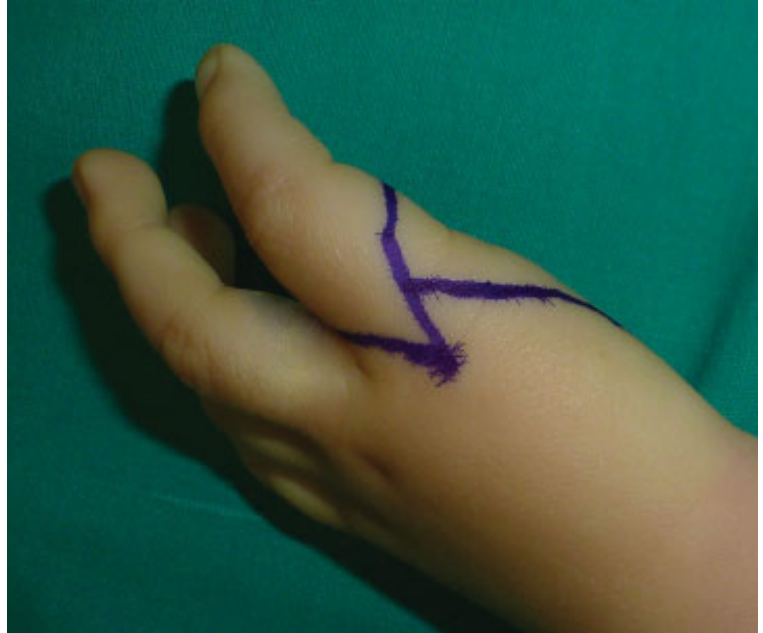

Fig. 2 Colgajo de base y vértice proximal en el dorso del índice, por lateral y volar alcanza la región media-distal de la F1 del índice.

venas dorsales (-Fig. $\mathbf{3}$ ), siempre una es de buen calibre. En nuestra práctica, siempre se logra un buen plano entre las venas y el aparato extensor, y deben ser aisladas para los pasos que vienen. Por volar la elevación del colgajo exige que llegue a nivel del 3er MTC, para lograr identificar claramente los paquetes neurovasculares del dedo índice y medio. Sin duda que esa elevación de colgajos producirá sufrimiento isquémico, pero nunca hemos visto necrosis de los mismos.

3. Disección de estructuras neurovasculares. Con frecuencia es posible identificar los dos paquetes neurovasculares del índice, pero en casos de hipoplasias muy severas, la arteria del lado radial no está presente. Al contrario, la arteria del lado cubital, siempre lo estará. En ambos casos, cuando se diseca la arteria del lado cubital, la división que va al dedo medio, debe ser ligada y cortada ( - Fig. 4). Para

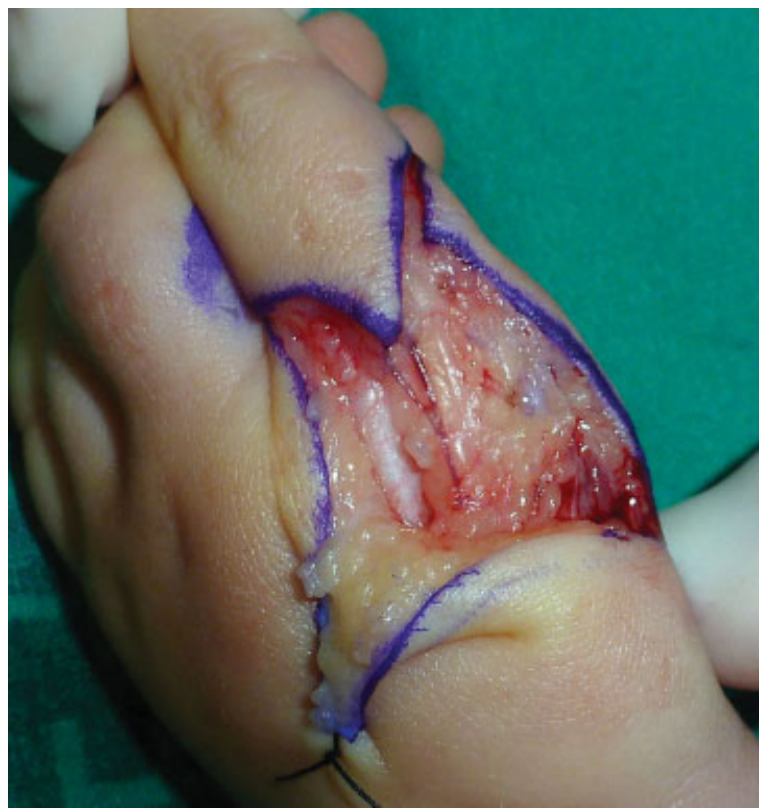

Fig. 3 Disección de la red venosa. Puede haber una o dos venas dorsales importantes. 


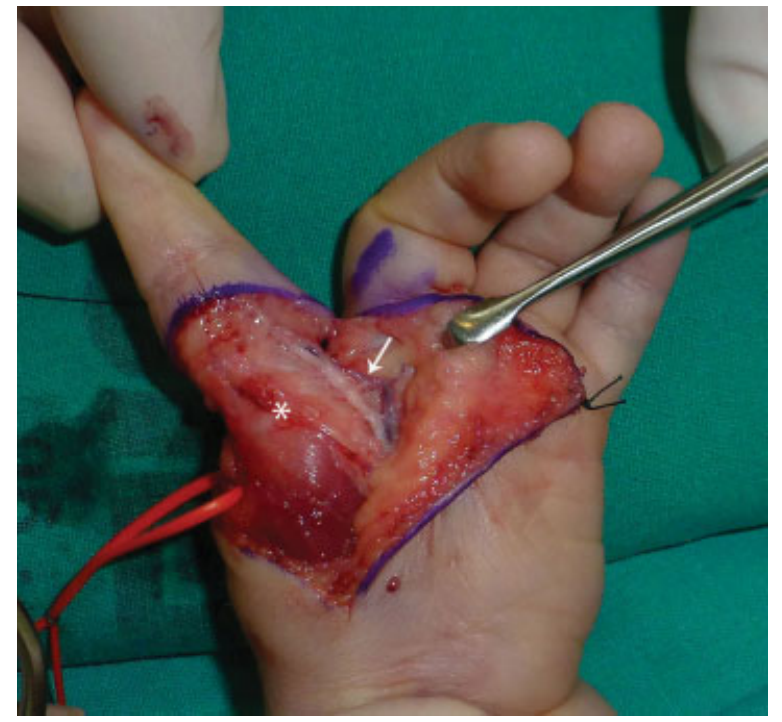

Fig. 4 Disección de paquetes neurovasculares. El asterisco muestra la arteria radial. La flecha muestra el nervio y la arteria cubital del índice.

la disección de los nervios, no hay problemas en disecar el del lado radial cuando está presente, pero en el caso del nervio colateral cubital, en ocasiones se debe hacer una intra neuro disección, y separarlo del nervio que va al dedo medio. No es difícil hacerlo. Para ambas estructuras la disección debe alcanzar un nivel al menos de hasta la mitad del MTC para lograr un transporte sin tensión.

4. Disección de los primeros músculos intersoeos. Esos músculos serán de mayor o menor tamaño dependiendo de la severidad de la hipoplasia, y que pueden condicionar la función de la transferencia. La disección de ambos músculos se hace desde distal a proximal, separándolos del MTC, en forma extraperiótica (-Fig. 5). Hay que realizar una cuidadosa sección de los tendones de esos músculos a nivel de la MTCF, cuidando de no dañar la

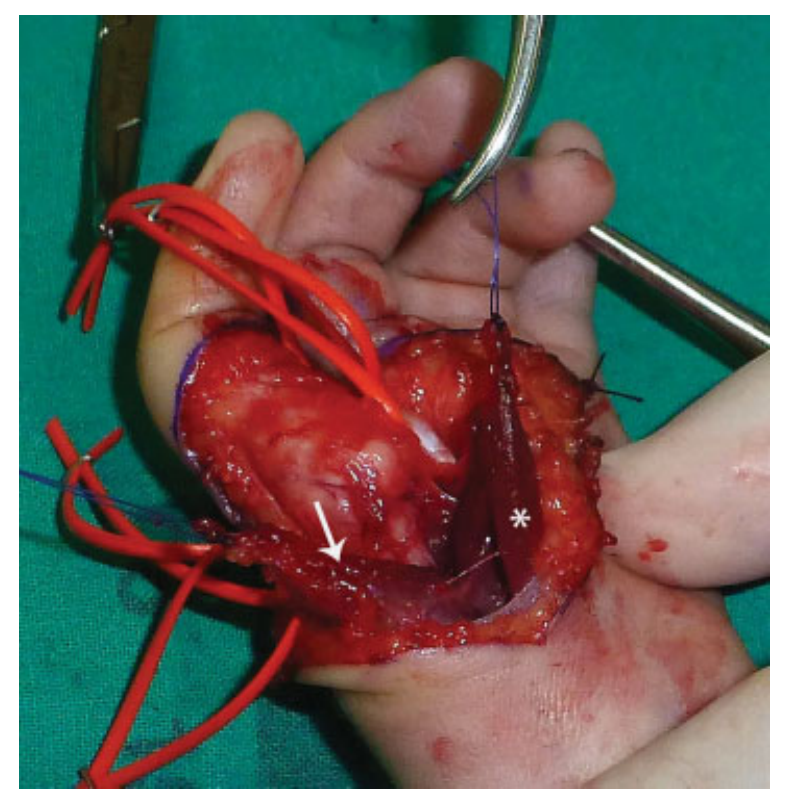

Fig. 5 Disección del musculo interóseo palmar (asterisco) y el musculo interóseo dorsal (flecha).

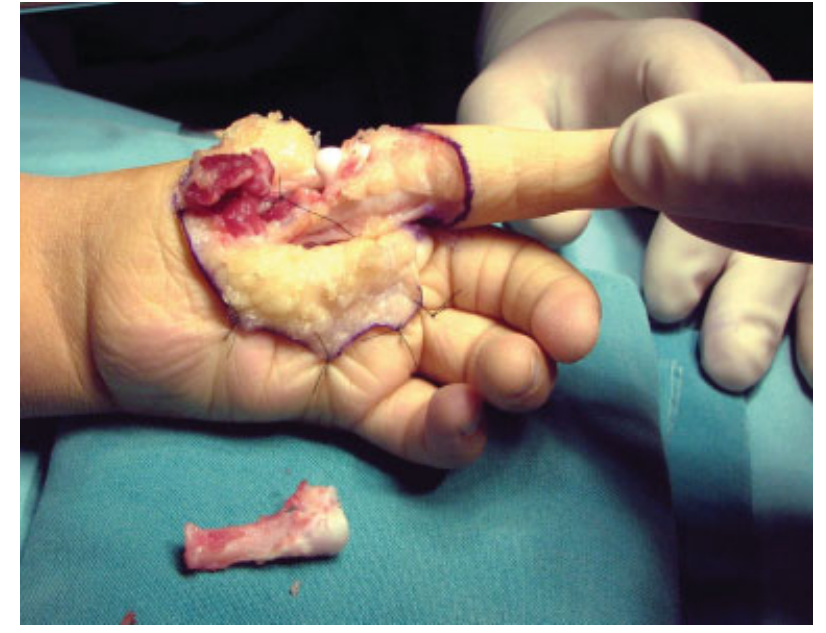

Fig. 6 Resección ósea de segundo MTC. El corte distal se realiza a nivel de la fisis.

cápsula articular. La disección se continúa a proximal, hasta la base del MTC, cuidando de respetar los ramos motores de esos músculos que se observan siempre a su nivel proximal.

5. Disección de aparato extensor y flexor. La disección del aparto extensor incluye la identificación de los dos extensores, para separarlos del MTC, y a nivel distal, lograr ver bien la separación entre este y los intrínsicos (importante al momento de la reinserción de los músculos interóseos). La identificación del flexor del índice, se limita a identificar la polea A y a cortarla.

6. Resección osea del MTC. En una mano normal la longitud del pulgar llegará aproximadamente al nivel de la F1 del índice, no más allá de la IFP. Por ello se debe resecar casi todo el 2do MTC. La resección distal se hace a nivel de la placa de crecimiento, lo que se facilita identificándola con una aguja hipodérmica y luego se corta con el uso de un bisturí. A proximal la resección se hace a nivel de la base con ayuda de una sierra (-Fig. 6). Algo que puede ayudar el nivel de corte, es resecar la longitud ósea que equivale a lo que mide el índice desde su ápice hasta la IFP.

7. Transporte digital. Ahora lo que queda es cortar la unión intermetacarpiana entre el 2do y 3er MTC, y separar los músculos intrínsicos que van al dedo medio. Así, el índice puede ser transportado a nivel de la base del 2do MTC. En esa maniobra, el digito debe quedar en una abducción de $45^{\circ}$, y pronado en $90^{\circ}$, de manera que el nuevo pulgar quede haciendo una pinza termino-terminal con el dedo medio (-Fig. 7). Algunas técnicas mencionan que esa pronación deber ser de entre 120 y $150^{\circ}$, sin embargo, no hay que olvidar que la nueva CMTC del nuevo pulgar, no queda con movilidad mulpiplanar, lo que puede hacer que ese pulgar quede fijo en un plano muy anterior.

8. Fijación de la cabeza del metacarpiano. Creemos que este es uno de los pasos más importantes de la cirugía, y hay que hacerlo teniendo en consideración 3 conceptos. El primero es que la cabeza del MTC se coloca sobre la base del MTC, ni delante ni detrás de este. Segundo, la MTCF del índice, se hiperextiende para ser fijada, de manera que la superficie 


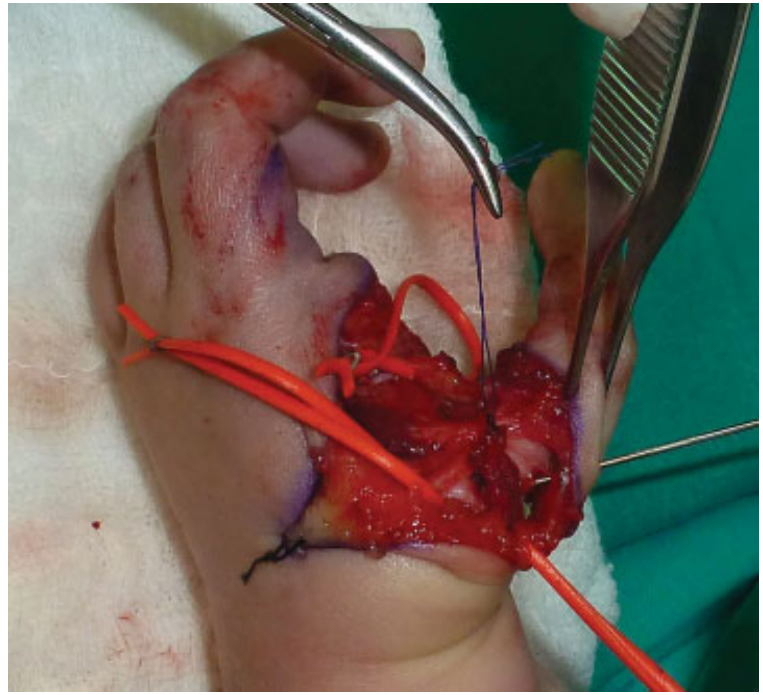

Fig. 7 El índice se ha colocado en su nueva posición, ayudado por una aguja de Kirchner. La pinza de Kelly tiene tomado un punto que guía la inserción del musculo interóseo dorsal.

articular de la cabeza del MTC mira a volar y la base de la cabeza, mira a dorsal. Eso es para evitar la hiperextensión del nuevo pulgar. Y tercero, la estabilización se hace con puntos transfixiantes en superficies cartilaginosa y ósea, con material no absorbible. Esos puntos son pasados en forma separadas antes de colocar en posición la nueva articulación, para facilitar la colocación de los mismos. En ese momento, y antes de fijar los puntos, nosotros pasamos una aguja de kirschner en forma retrograda como sugiere Kosin,7 y con ayuda de esta fijación podemos manejar la posición de la articulación es todos los planos. Terminamos de pasar la aguja para anclarla en la base del MTC, y terminamos de fijar las suturas.

9. Tranferencia de tendones. En la base del nuevo pulgar, hay que identificar las bandas laterales del retináculo extensor, así el primer interóseo dorsal es fijado en el lado radial del retináculo (nuevo "abductor pollicis"), y el primer interóseo palmar se fija al lado cubital del retináculo (nuevo "adductor pollicis") (-Fig. 7). Nosotros normalmente hacemos una sutura-plicatura del tendón extensor para acortar su longitud. Algunos proponen además disecar el tendón extensor propio del índice (cuando está presente), y suturarlo en lado radial del retináculo extensor para aumentar el efecto abductor.

10. Cierre de la piel. En ese momento se suelta la isquemia para observar la vitalidad de los tejidos. En nuestra experiencia, no hemos tenido complicaciones con isquemias del índice transferido. Se trasladan los colgajos y se procede al cierre y sutura de la piel. Así el vértice dorsal del índice es suturado en la parte más proximal de la incisión palmar, lo que ayuda a determinar también la posición de rotación en que quedará el nuevo pulgar. Otro aspecto importante es que la piel volar es colocada de manera que quede cubriendo todo lo que será el primer espacio, evitando al máximo que ese espacio quede con suturas, ya que ellas pueden producir cicatrices retráctiles ( $\mathbf{F i g}$. 8). En ocasiones,

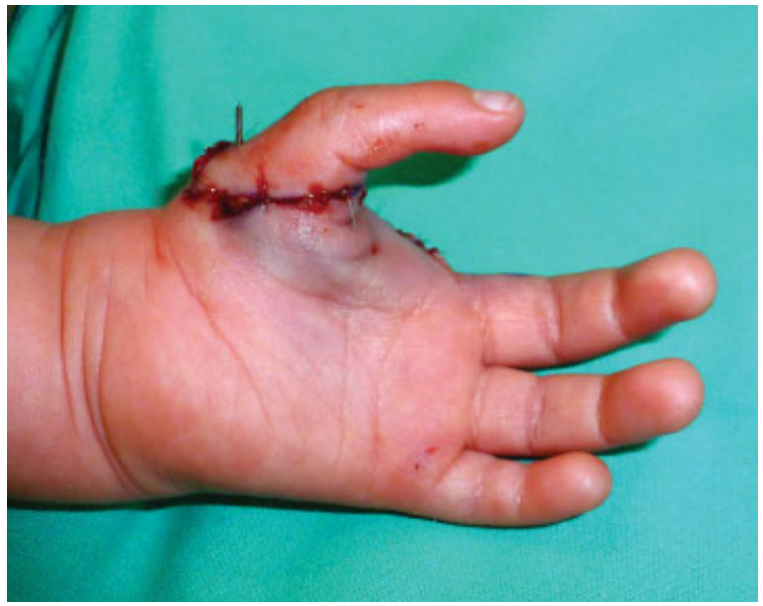

Fig. 8 Transposición de los colgajos y se sutura la piel. La aguja se deja por 4 semanas.

queda algo de piel redundante, que debe ser resecada y nunca hemos necesitado injerto de piel para el cierre.

11. Cuidados postoperatorios. Se coloca una valva de yeso por 4 semanas, y entonces es removida junto a la aguja de kirschner. A eso le adicionamos una órtesis termoplástica, que es removida a la sexta semana de la cirugía, y se inician las actividades de pinza. Tanto para el manejo y confección de la órtesis, como para las actividades de pinza, es fundamental la participación de la Terapia Ocupacional. En general, le pedimos a los padres que el niño siga usando la órtesis nocturna por unos 3 meses.

Esa técnica reporta escasas complicaciones derivadas del acto quirúrgico. Es posible ver sufrimientos de piel de los colgajos y cicatrices retráctiles. Otras complicaciones se refieren a deficiencias en la función, como limitaciones a la oposición o extensión del nuevo pulgar, que pudieran requerir de cirugías. ${ }^{8}$ Complicaciones mayores, como fallas arteriales y venosas son raras. Buck-Gramcko reporta 12 lesiones arteriales en 460 pulgarizaciones. ${ }^{9}$ En nuestra experiencia de 10 años, habiendo realizado 27 pulgarizaciones, nunca hemos tenido falla arterial y venosa.

\section{Factores asociados al éxito de la cirugía}

El principal factor que se asocia a la calidad de la transferencia es la calidad del índice a transferir. Es muy importante ver la hipoplasia que puede asociarse al índice, al momento de planificar la transferencia. En "buen índice," se asocia a buenos tendones y a una buena musculatura intrínsica, lo que se relaciona con la capacidad de movimientos que tendrá el nuevo pulgar., ${ }^{6-12}$ Por esa razón, la mejor indicación de pulgarizar el índice, será en un paciente con hipoplasia aislada del pulgar, no asociado a alteraciones en el radio, o a índices rígidos ${ }^{7}$ (-Figs. 9 y 10).

Otro factor es la edad en que se hace la transferencia. Si bien la plasticidad cerebral permite por muchos años adecuarse a una pinza digito-pulgar (lo vemos en transplantes de mano, transferencias de ortejos a pulgar), ${ }^{13-15}$ pulgarizar el índice a temprana edad mejora las habilidades que el niño pueda 


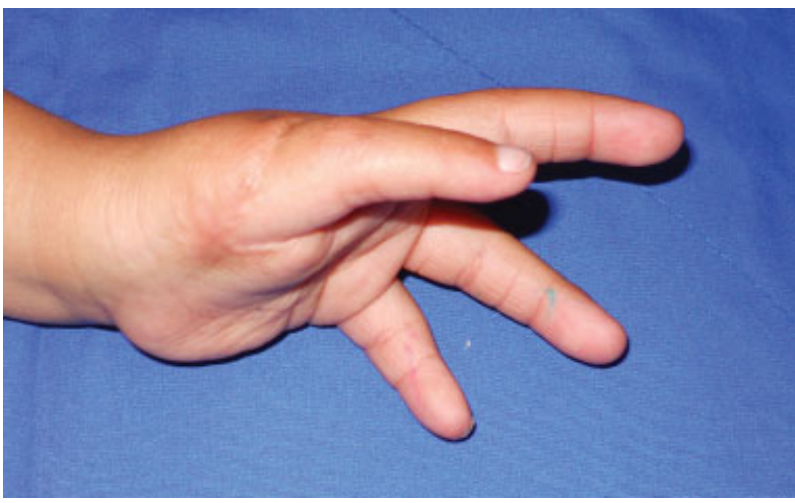

Fig. 9 Resultado a los 18 meses de la operación. Se observa una adecuada posición del pulgar.

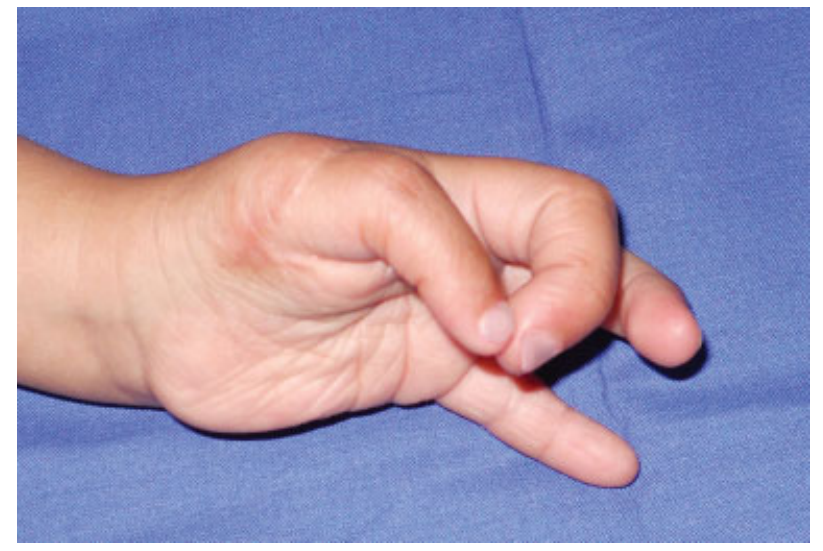

Fig. 10 Resultado a los 18 meses de la operación. Logra una buena pinza termino-terminal.

realizar con su nueva pinza. Lo ideal es hacerlo al año de edad. Así, tendremos estructuras neurovasculares adecuadas para la cirugía, y el niño tiene tiempo para adquirir sus habilidades en la etapa preescolar.

También se ha mencionado como factor pronóstico la técnica quirúrgica que se refiere a la posición y anclaje óseo que se logre, junto a las transferencias musculares, y alineamiento de tendones. Todo eso influye en la posición y función que logra la pulgarización. Ese aspecto está en estrecha relación con la experiencia que tenga el cirujano y por lo mismo se aconseja que quede en manos de equipos de trabajo que concentren ese tipo de patologías.

\section{Discusión}

La pulgarización del índice es la técnica de elección para la reconstrucción de la pinza en hipoplasias de pulgar. La pinza lograda definitivamente entrega una mano que puede hacer pinza y tomar objetos grandes. La pinza fina también mejora.

La fuerza de agarre que se logra, según distintas series, es de un $63 \%$ a un $75 \%$, y la fuerza de pinza es de un $56 \%$ a un $65 \%$, lo que estaría en relación con la calidad del índice y la técnica empleada. ${ }^{12,16-18}$

La pulgarización del índice es una técnica demandante, con pasos bien establecidos, y en manos entrenadas entrega una mano con una buena pinza de agarre para objetos grandes principalmente. El tiempo óptimo para realizar es alrededor del año de vida, ya que el niño puede incorporar la nueva mano con más facilidad, logrando mayores habilidades.

\section{Bibliografía}

1 Hilgenfeldt 0 . Operative Daumenersatz um Beseitigung von Grifstorangen bei Fingerverlusten. FerdinandEnke Verlag, Stuttgart, 1950

2 Gosset J. La pollicisation de l'index; technique chirurgicale. J Chir (Paris) 1949;65(7-8):403-411

3 Littler JW. The neurovascular pedicle method of digital transposition for reconstruction of the thumb. Plast Reconstr Surg (1946) 1953;12(05):303-319

4 Buck-Gramcko D. Pollicization of the index finger. Method and results in aplasia and hypoplasia of the thumb. J Bone Joint Surg Am 1971;53(08):1605-1617

5 Blauth W. [The hypoplastic thumb]. Arch Orthop Unfallchir 1967; 62(03):225-246

6 Manske PR, McCaroll HR Jr. Index finger pollicization for a congenitally absent or nonfunctioning thumb. J Hand Surg Am 1985;10(05):606-613

7 Kozin SH. Pollicization: the concept, technical details, and outcome. Clin Orthop Surg 2012;4(01):18-35

8 Goldfarb CA, Monroe E, Steffen J, Manske PR. Incidence and treatment of complications, suboptimal outcomes, and functional deficiencies after pollicization. J Hand Surg Am 2009;34(07):1291-1297

9 Buck-Gramcko D. Complications and bad results in pollicization of the index finger (in congenital cases). Ann Chir Main Memb Super 1991;10(06):506-512

10 Kozin SH, Weiss AA, Webber JB, Betz RR, Clancy M, Steel HH. Index finger pollicization for congenital aplasia or hypoplasia of the thumb. J Hand Surg Am 1992;17(05):880-884

11 Buck-Gramcko D. Pollicization of the index finger. Method and results in aplasia and hypoplasia of the thumb. J Bone Joint Surg Am 1971;53(08):1605-1617

12 Vekris MD, Beris AE, Lykissas MG, Soucacos PN. Index finger pollicization in the treatment of congenitally deficient thumb. Ann Plast Surg 2011;66(02):137-142

13 Anastakis DJ, Malessy MJ, Chen R, Davis KD, Mikulis D. Cortical plasticity following nerve transfer in the upper extremity. Hand Clin 2008;24(04):425-444, vi-vii

14 Chen R, Anastakis DJ, Haywood CT, Mikulis DJ, Manktelow RT. Plasticity of the human motor system following muscle reconstruction: a magnetic stimulation and functional magnetic resonance imaging study. Clin Neurophysiol 2003;114(12):2434-2446

15 Silva AC, Rasey SK, Wu X, Wall JT. Initial cortical reactions to injury of the median and radial nerves to the hands of adult primates. J Comp Neurol 1996;366(04):700-716

16 Percival NJ, Sykes PJ, Chandraprakasam T. A method of assessment of pollicisation. J Hand Surg [Br] 1991;16(02):141-143

17 Manske PR, Rotman MB, Dailey LA. Long-term functional results after pollicization for the congenitally deficient thumb. J Hand Surg Am 1992;17(06):1064-1072

18 Roper BA, Turnbull TJ. Functional assessment after pollicisation. J Hand Surg [Br] 1986;11(03):399-403 